\title{
Femtosecond Laser Pulse Driven Caustic Spin Wave Beams
}

\author{
S. Muralidhar $\odot,{ }^{1,}{ }^{*}$ R. Khymyn $\odot,{ }^{1}$ A. A. Awad $\odot,{ }^{1}$ A. Alemán, ${ }^{1}$ D. Hanstorp, ${ }^{1}$ and J. Åkerman $\oplus^{1,2, \dagger}$ \\ ${ }^{1}$ Physics Department, University of Gothenburg, 41296 Gothenburg, Sweden \\ ${ }^{2}$ Materials and Nano Physics, School of Engineering Sciences, KTH Royal Institute of Technology, \\ Electrum 229, 16440 Kista, Sweden
}

(Received 16 June 2020; revised 22 September 2020; accepted 7 January 2021; published 22 January 2021)

\begin{abstract}
Controlling the directionality of spin waves is a key ingredient in wave-based computing methods such as magnonics. In this Letter, we demonstrate this particular aspect by using an all-optical pointlike source of continuous spin waves based on frequency comb rapid demagnetization. The emitted spin waves contain a range of $k$ vectors and by detuning the applied magnetic field slightly off the ferromagnetic resonance (FMR), we observe $\mathrm{X}$-shaped caustic spin wave patterns at $70^{\circ}$ propagation angles as predicted by theory. When the harmonic of the light source approaches the FMR, the caustic pattern gives way to uniaxial spin wave propagation perpendicular to the in-plane component of the applied field. This field-controlled propagation pattern and directionality of optically emitted short-wavelength spin waves provide additional degrees of freedom when designing magnonic devices.
\end{abstract}

DOI: 10.1103/PhysRevLett.126.037204

Caustics are widely known from optics as the envelopes of light rays refracted by curved surfaces or objects [1,2]. The notion, however, can be easily extended to any propagation medium whose anisotropy creates regions with enhanced amplitude of the wave field [3]. The phenomenon of caustic patterns encompasses an extraordinarily wide range of fields in physics, from understanding the physics of the Universe, its creation, and dark matter dynamics [4] to studying light patterns at the bottom of a pool due to surface curvature variations.

The important feature, known from the simple paraxial case in geometrical optics, is the structural stability of the caustics, which imply small fuzziness of the caustic beam under variation of the scattering surface curvature [2]. This opens a way to create nondiffractive beams in anisotropic continuous media, i.e., channeled in a certain direction, e.g., focusing of propagating phonons [5-7]. Similar effects have also been observed for spin waves (SWs) in magnetic materials, where the anisotropic properties can furthermore be readily modified by the strength and direction of the applied magnetic field. Early experiments used a pointlike defect to demonstrate the unidirectional excitation of magnetostatic surface waves and the propagation of backward volume magnetostatic waves in two preferential directions due to the noncollinearity of their phase and group velocities [8]. Further advances made possible the

Published by the American Physical Society under the terms of the Creative Commons Attribution 4.0 International license. Further distribution of this work must maintain attribution to the author(s) and the published article's title, journal citation, and DOI. Funded by Bibsam. emission of caustic patterns by patterned SW waveguides connected to extended thin films, both insulating (YIG) [9] and metallic (NiFe) [10], where the SWs were excited by microwave antennas. Caustic beams can also be emitted from a collapsing bullet mode [11] or from spin wave edge modes in Heusler compounds [12]. Detailed theoretical analyses of SW caustics is now available for ferromagnetic metals [13], insulators [14], and antiferromagnetic thin films [15]. Key application of caustics is in the field of magnonics where it can, e.g., be utilized as a potential signal splitter [16], frequency-division multiplexer [17], and demultiplexer [18] in magnonic networks. Additionally, the focusing effects of caustic beams can be utilized to minimize the diffraction spread of long-distance propagating SWs [19].

While all the above experiments on SW caustics used rf antennas for the initial SW excitation, recent interest in exciting SWs using femtosecond lasers has rapidly increased [20-34]. However, no such optical SW excitation studies have shown any sign of caustics. This is likely due to earlier studies not combining small enough laser spots (for efficient excitation of a wide range of $k$ vectors) with a sufficiently high repetition rate excitation for strong caustics to form and be observed. Here we overcome this limitation by focusing a $1 \mathrm{GHz}$ repetition rate femtosecond laser comb down to submicron spot size capable of sustaining continuous SWs $[30,32,35,36]$. The emitted SWs contain a wide range of $k$ vectors and, by slight detuning of the applied magnetic field off the ferromagnetic resonance (FMR), we observe X-shaped caustic SW patterns at around $70^{\circ}$ angles as predicted by theory. When the harmonic of the light source approaches the FMR, the caustic pattern gives a way to uniaxial SW 


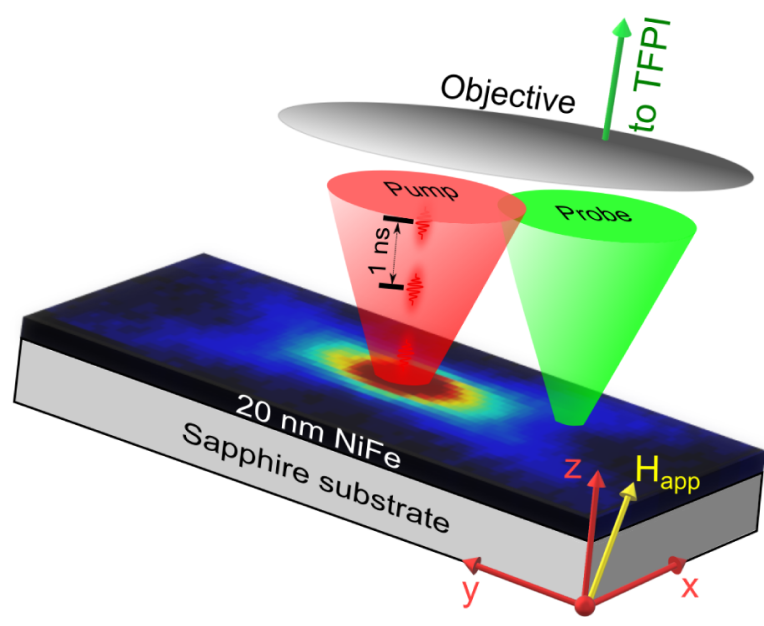

FIG. 1. Schematic of the experiment. A $20 \mathrm{~nm} \mathrm{NiFe}$ film on a transparent sapphire substrate is pumped with a $1 \mathrm{GHz}-$ pulsed femtosecond laser (120 fs) at a wavelength of $816 \mathrm{~nm}$ (red) and probed by a continuous single-mode $532 \mathrm{~nm}$ Brillouin light scattering laser (green). The magnetic field $H_{\text {app }}$ is applied at an oblique angle of $82^{\circ}$ out of plane with its in-plane component along the $y$ axis. Backscattered photons (green arrow) are analyzed using a six-pass tandem Fabry-Perot interferometer (TFPI).

propagation perpendicular to the in-plane component of the applied field. This field-controlled propagation pattern and directionality of optically excited short-wavelength SWs provide additional degrees of freedom when designing magnonic devices without any requirements for additional patterning for such functions.

Experimental method.-Figure 1 shows the schematic of the experiment. A more in-depth description can be found in Ref. [36]. Briefly, the pump-probe experiment uses a mode-locked femtosecond laser operating at $816 \mathrm{~nm}$ and a repetition rate of $1 \mathrm{GHz}$ with $120 \mathrm{fs}$ long pulses to induce rapid demagnetization. Each laser pulse instantaneously increases the temperature of the electron gas in the thin metallic film. The thermal energy of the electrons is then rapidly transferred to the magnetic subsystem, creating a rapid demagnetization of the irradiated region and hence a rapid pulse of the local effective magnetic field. The periodic modulation of the demagnetizing field excites magnons [26] with frequencies that are a multiple of the $1 \mathrm{GHz}$ repetition rate of the pump-laser pulses [32].

The excited SWs are studied using Brillouin light scattering (BLS) microscopy. A $532 \mathrm{~nm}$ continuous wave laser is used to probe the system where the photons are inelastically scattered by the magnons and are analyzed using a tandem Fabry-Perot interferometer [37]. Both the pump and probe lasers are focused close to their respective diffraction limits onto the sample using a high numerical aperture $(\mathrm{NA}=0.75)$ objective to allow excitation and detection of magnons with high wave vectors up to $k \simeq 10 \mathrm{rad} / \mu \mathrm{m}$. The setup is also equipped with a pair

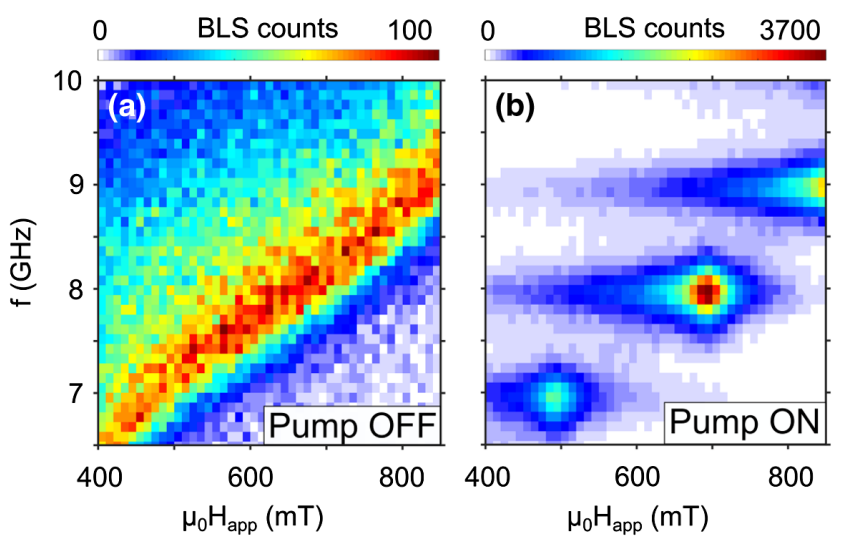

FIG. 2. Field vs frequency plot of the SW amplitude for NiFe $20 \mathrm{~nm}$ thin film showing (a) thermal magnons and (b) stimulated magnons by a pump laser with a fluence of $3.6 \mathrm{~mJ} / \mathrm{cm}^{2}$.

of galvo mirrors and lenses providing the means to measure the SW amplitude at a relative distance $l$ between the pump and the probe beam spots.

The sample under study was a $20 \mathrm{~nm}$ thick polycrystalline $\mathrm{NiFe}$ (permalloy) thin film deposited on a sapphire substrate. The permalloy film was coated with $2 \mathrm{~nm}$ of dielectric $\mathrm{SiOx}$ to prevent surface oxidation. The sample was placed in an oblique ( $82^{\circ}$ out of plane) magnetic field $\left(H_{\text {app }}\right)$. The oblique field is chosen to provide compatibility with arrays of spin-Hall nano-oscillators (SHNOs), where, e.g., mutual synchronization can be optimized by the field angle [38-40], which would allow for studying the effect of SW caustics in SHNOs.

Results.-Figure 2 shows the BLS spectra obtained for a small range of field from 400 to $850 \mathrm{mT}$ with the pump [Fig. 2(a)] off and [Fig. 2(b)] on. When the pump is off, the spectrum corresponds to the thermal magnons, which show a sharp field-dependent cutoff toward lower frequencies, corresponding to the SW gap, and a more gradual decrease toward higher fields due to the dropoff in wave vector resolution of the BLS. When we turn on the pump laser, a series of peaks, corresponding to the harmonics of the $1 \mathrm{GHz}$ repetition rate, appear, showing how additional magnons are strongly excited at frequencies matching the repetition rate. The peaks are approximately located at the onset of the broadband ferromagnetic resonance signal. Each individual harmonic mode at $7-9 \mathrm{GHz}$ in Fig. 2(b) from the peak are extended toward lower fields and drastically drop in intensity at higher fields. At low fields, these modes correspond to propagating SWs with high $k$ vector; at higher fields, the modes fall into the $\mathrm{SW}$ band gap and are evanescent in time and space (nonresonant, forced oscillations). The SW band gap in this range of fields takes place at relatively low wave vectors, far below the maximum resolution of the BLS.

To determine the spatial extent of the SW modes at different applied field strength, we collected 11 SW intensity area maps for fields starting from 475 to 


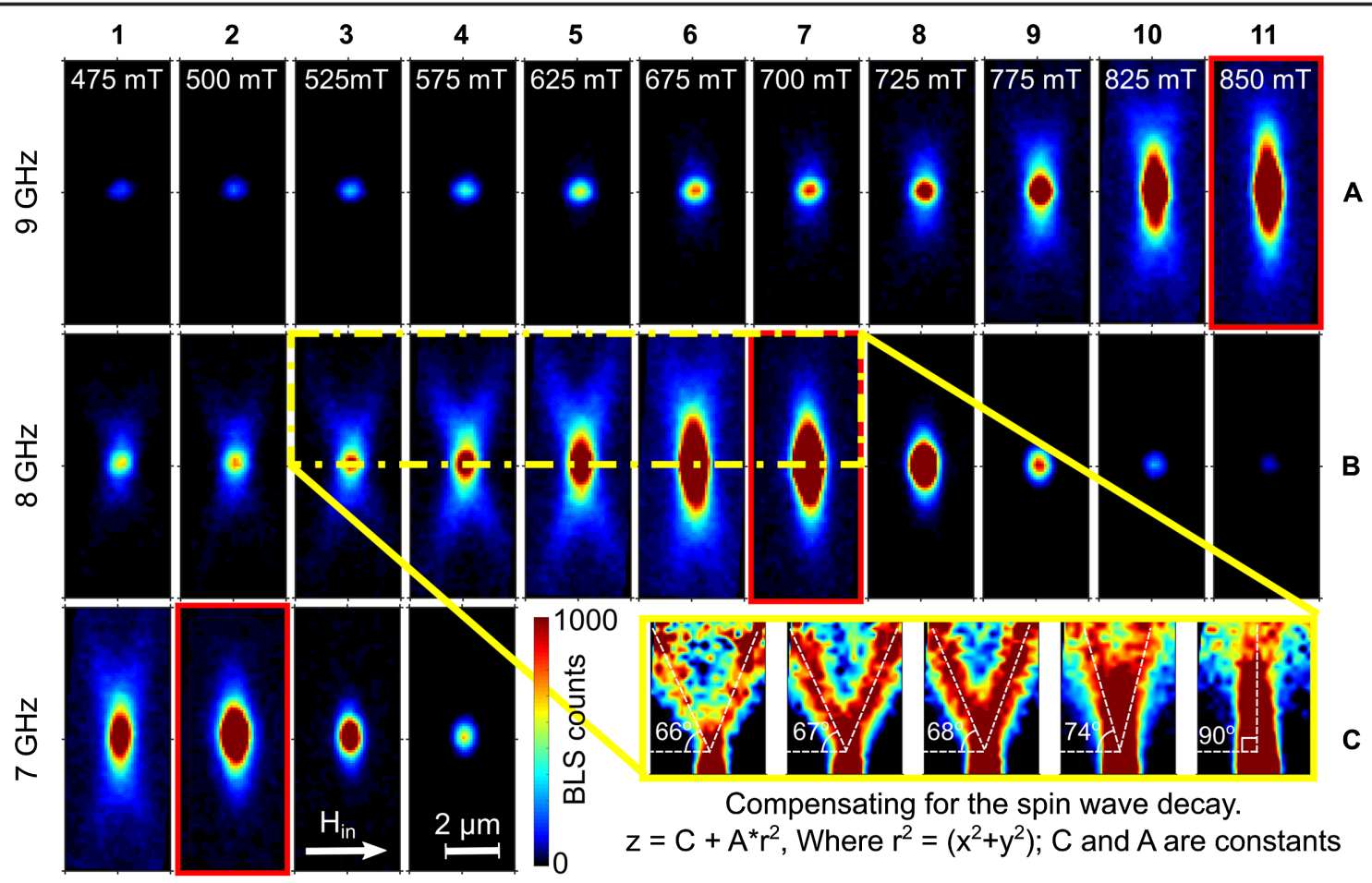

FIG. 3. Spatial profiles of NiFe thin film measured in an area $4 \times 10 \mu \mathrm{m}^{2}$ for 11 different field values. The area maps are plotted for (A) $7 \mathrm{GHz}$, (B) $8 \mathrm{GHz}$ and (C) $9 \mathrm{GHz}$ modes. The red outlined maps correspond to the maps plotted for the peak SW amplitude for individual modes in Fig. 2(b). The color scale is the same for all plots. Inset: contains propagation characteristics of the $8 \mathrm{GHz}$ mode for five field values (B3)-(B7) giving an estimate of the caustic angle. The color maps in the inset are compensated for the SW decay.

$850 \mathrm{mT}$ (Fig. 3). Since the BLS microscope separates counts directly in the frequency domain, the three modes (7-9 GHz) can be separately plotted by selecting the specific channels corresponding to individual frequencies. All subplots use the same color scale, shown at the bottom of the figure. The spatial profiles highlighted by red outline [(a11), (b7), and (c2)] correspond to the maximum SW intensity on the field sweep plot [Fig. 2(b)]. They clearly show a strong straight extension along the direction perpendicular to the in-plane component of the applied field $\left(H_{\text {in }}\right)$. At higher fields, the spatial profile transforms into a localized spot, which eventually fades away as the now evanescent SW mode moves deeper into the SW gap.

Reducing the field, on the other hand, dramatically changes the spatial profile at all three frequencies. We here focus on the $8 \mathrm{GHz}$ mode where fields lower than $700 \mathrm{mT}$ show a prominent caustic behavior with a propagation angle that decreases with field (i.e., with increasing $k$ vector); this can be seen in the highlighted yellow section. Here the dashed white lines are a guide to the eye to follow how the SW beam pattern changes from perpendicular propagation with respect to the in-plane component of the applied field to a propagation angle of $\sim 66^{\circ}$ with decreasing field. The weaker SW intensity in Figs. 3(b1) and 3(b2) are due the decreasing efficiency of both the excitation and the BLS detection at higher wave vector. The same overall behavior is found at 7 and $9 \mathrm{GHz}$.
Theoretical analysis.-It is convenient to start with considering the anisotropic properties in Fourier space of a continuous medium. A small source with the characteristic size $r$ can emit waves with all wave vectors $(\mathbf{k})$ up to $\sim 1 / r$ and group velocities $\left(\mathbf{v}_{\text {gr }}\right)$, defined by a dispersion relation of the media as $\mathbf{v}_{\mathrm{gr}}=2 \pi \nabla f(\mathbf{k})$. Thus, if the surface of the emitted frequency $f_{0}(\mathbf{k})$ (isofrequency surface) contains "flat" regions, all waves with wave vectors in this region have the same direction of $\mathbf{v}_{\mathrm{gr}}$, which leads to the formation of high-intensity unidirectional beams [41,42]. The analysis of caustic SW beams in a thin magnetic film is described in detail in [9], which we follow here. First, we calculate the magnon dispersion following a simplified version of Eq. (45) in [43]. For in-plane SWs, this reads

$$
\begin{aligned}
f= & \frac{\gamma \mu_{0}}{2 \pi} \sqrt{\left(H_{\mathrm{int}}+M_{s} l_{\mathrm{ex}}^{2} k^{2}\right)\left(H_{\mathrm{int}}+M_{s} l_{\mathrm{ex}}^{2} k^{2}+M_{s} F_{0}\right)}, \\
F_{0}= & P_{0}+\cos ^{2} \theta_{\mathrm{int}}\left\{1-P_{0}\left(1+\cos ^{2} \phi\right)\right. \\
& \left.+M_{s}\left[P_{0}\left(1-P_{0}\right) \sin ^{2} \phi\right] /\left(H_{\mathrm{int}}+M_{s} l_{\mathrm{ex}}^{2} k^{2}\right)\right\},
\end{aligned}
$$

where $P_{0}=1-[1-\exp (-k L)], l_{\mathrm{ex}}=\sqrt{2 A /\left(\mu_{0} M_{s}^{2}\right)}$, and $H_{\text {int }}$ and $\theta_{\text {int }}$ define the value and out of plane (OOP) angle of the internal field; these can be found using the solution of the magnetostatic problem [e.g., Eq. (2.3) in [44] ] 


$$
\begin{gathered}
H_{\text {app }} \cos \theta_{\text {app }}=H_{\text {int }} \cos \theta_{\text {int }}, \\
H_{\text {app }} \sin \theta_{\text {app }}=\left(H_{\text {int }}+M_{s}\right) \sin \theta_{\text {int }},
\end{gathered}
$$

where $H_{\text {app }}$ denotes the external magnetic field applied at an OOP angle $\theta_{\text {app }}$.

The following parameters were used: saturation magnetization $\mu_{0} M_{s}=0.8 \mathrm{~T}$, exchange stiffness $A=11.3 \mathrm{pJ} / \mathrm{m}$, film thickness $L=20 \mathrm{~nm}$, and $\theta_{\text {app }}=82^{\circ}$. The direction between the in-plane component of the field $H_{\text {in }}$ and the wave vector $\mathbf{k}$ is defined by $\phi$. The frequency of magnons vs wave vector components $k_{x}=k \sin \phi$ and $k_{y}=k \cos \phi$ is shown by contour plots in Fig. 4.

In the next step, we extract the isofrequency line $k_{x}=g\left(f, k_{y}\right)$ for the frequency of excitation $f=8 \mathrm{GHz}$, which is shown by the red line in Fig. 4. The condition for a caustic beam is a flat region on this curve, namely,

$$
\partial^{2} g\left(f, k_{y}\right) / \partial k_{y}^{2}=0
$$

from which we can extract the wave vectors of caustic beams $\mathbf{k}_{c}$, which are shown in Figs. 4(a) and 4(b) by the green dots. The group velocities and, thus, the propagation directions of the caustic beams are perpendicular to the isofrequency line at these points, which can be written as $\pi / 2-\phi_{c}=\arctan \left(\partial g\left(f, k_{y}\right) / \partial k_{y}\right)$. The directions of the propagation are shown by green arrows.
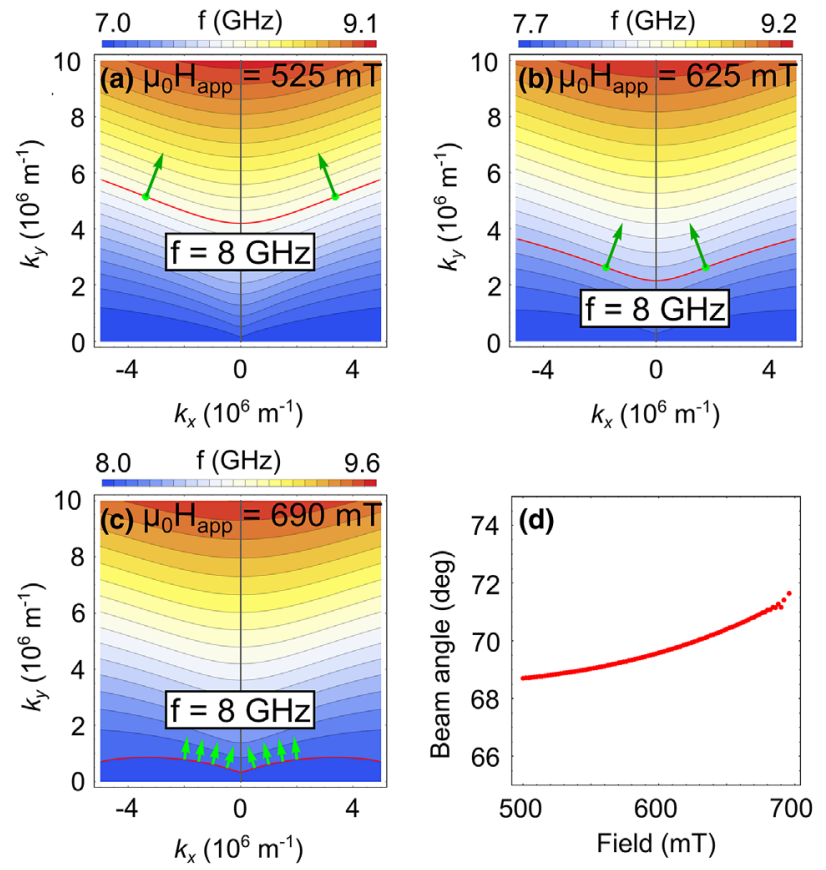

FIG. 4. (a)-(c) Isofrequency curves for $8 \mathrm{GHz}$ for different applied fields. Caustic points defined by Eq. (4) are shown by green dots, while directions of the caustic beams are shown by the darker arrows. (c) The preferred direction of the emitted SWs for the high field, where the caustic X-shape pattern does not exist. (d) Caustic beam angle vs applied magnetic field.
With increasing field, the wave vector of the caustic beam decreases [see Fig. 4(d)], until at some point $k_{x} \rightarrow 0$. At this point, the condition in Eq. (4) can no longer be satisfied for the particular isofrequency curve, and, as a consequence, the caustic beam no longer exists. Instead the isofrequency shows a nonzero second derivative everywhere, as illustrated by the green arrows in Fig. 4(c). For our choice of parameters, this happens at $\mu_{0} H_{\text {app }} \simeq 0.69 \mathrm{~T}$. One can notice that the slope of isofrequency curve at high fields is quite small. Taking into account its symmetry for positive and negative $k_{x}$, this leads to the focusing of the emitted SWs primarily into the $y$ direction, as we observed above from the BLS spatial maps.

As one can notice from Fig. 4(d), the calculated angles of the caustic beams slightly differ from the experimental values, which can, e.g., be explained by the finite size of the excitation source, as observed in [18]. In addition, the caustic angles depend significantly on the magnetic parameters of the film, especially on the exchange constant $A$, since the caustic point is forming at the interplay between the dipolar and the exchange part of the spin wave spectrum. For example, a reduction of $A$ leads to an increase of the slope in Fig. 4(d) and hence to a better matching with experimental results. Such a reduction of the exchange constant might be thermal due to the intense laser irradiation [45].

In conclusion, we have demonstrated the generation of field-tunable caustic SW beams in $20 \mathrm{~nm}$ NiFe films using an all-optical excitation mechanism based on a femtosecond laser comb. The spatial profiles and frequency content of the generated SWs were mapped out using scanning Brillouin light scattering microscopy. With a high repetition rate laser, we excite only those SWs whose frequency matches the harmonics of the fs laser $1 \mathrm{GHz}$ repetition rate. The implications of suppressing all other SW modes other than the one corresponding to the harmonic modes of the pump laser leads to the possibility of controlling the emission direction by tuning the magnetic field. Theoretically, we have calculated the angle of the caustic SW beams with reference to the in-plane component of the applied field by plotting the isofrequency lines and applying the condition for caustic beams. Experimentally, we have plotted the area maps of the SW amplitude at various magnetic field strength values and estimated approximate values of the caustic beam angles. Additionally, by tuning the field, we are able to change the propagation characteristics from localized at high fields, to strongly propagating in a single direction when the ferromagnetic resonance frequency matches the harmonics of pump laser, and to $\mathrm{X}$-shaped caustic patterns at lower fields for a particular SW mode. The highly tunable properties of the excited SWs can be utilized in the field of photomagnonics.

Early discussions with R. E. Camley are gratefully acknowledged. This work was partially supported by the 
Swedish Research Council (VR, Grant No. 2016-05980), the Knut and Alice Wallenberg foundation (KAW, Grant No. 2015-0060), and the Horizon 2020 Research and Innovation Programme (ERC Advanced Grant No. 835068 “TOPSPIN”).

*shreyas.muralidhar@physics.gu.se

†johan.akerman@physics.gu.se

[1] O. Stavroudis, The Optics of Rays, Wavefronts, and Caustics (Elsevier, New York, 2012), Vol. 38.

[2] J. F. Nye, Natural Focusing and Fine Structure of Light: Caustics and Wave Dislocations (CRC Press, Boca Raton, FL, 1999).

[3] Y. A. Kravtsov and Y. I. Orlov, Caustics, Catastrophes and Wave Fields (Springer Science \& Business Media, New York, 2012), Vol. 15.

[4] V. I. Arnold, S. F. Shandarin, and Y. B. Zeldovich, The large scale structure of the universe I. General properties. One-and two-dimensional models, Geophys. Astrophys. Fluid Dyn. 20, 111 (1982).

[5] B. Taylor, H. J. Maris, and C. Elbaum, Phonon Focusing in Solids, Phys. Rev. Lett. 23, 416 (1969).

[6] A. G. Every, Formation of phonon-focusing caustics in crystals, Phys. Rev. B 34, 2852 (1986).

[7] A. Maznev and A. Every, Formation of surface phonon focusing caustics in crystals, Solid State Commun. 97, 679 (1996).

[8] O. Büttner, M. Bauer, S. O. Demokritov, B. Hillebrands, Y. S. Kivshar, V. Grimalsky, Y. Rapoport, and A. N. Slavin, Linear and nonlinear diffraction of dipolar spin waves in yttrium iron garnet films observed by space- and timeresolved Brillouin light scattering, Phys. Rev. B 61, 11576 (2000).

[9] T. Schneider, A. A. Serga, A. V. Chumak, C. W. Sandweg, S. Trudel, S. Wolff, M. P. Kostylev, V. S. Tiberkevich, A. N. Slavin, and B. Hillebrands, Nondiffractive Subwavelength Wave Beams in a Medium with Externally Controlled Anisotropy, Phys. Rev. Lett. 104, 197203 (2010).

[10] V. E. Demidov, S. O. Demokritov, D. Birt, B. O'Gorman, M. Tsoi, and X. Li, Radiation of spin waves from the open end of a microscopic magnetic-film waveguide, Phys. Rev. B 80, 014429 (2009).

[11] M. P. Kostylev, A. A. Serga, and B. Hillebrands, Radiation of Caustic Beams from a Collapsing Bullet, Phys. Rev. Lett. 106, 134101 (2011).

[12] T. Sebastian, T. Brächer, P. Pirro, A. A. Serga, B. Hillebrands, T. Kubota, H. Naganuma, M. Oogane, and Y. Ando, Nonlinear Emission of Spin-Wave Caustics from an Edge Mode of a Microstructured $\mathrm{co}_{2} \mathrm{mn}_{0.6} \mathrm{fe}_{0.4} \mathrm{Si}$ Waveguide, Phys. Rev. Lett. 110, 067201 (2013).

[13] V. Veerakumar and R. E. Camley, Magnon focusing in thin ferromagnetic films, Phys. Rev. B 74, 214401 (2006).

[14] V. Veerakumar and R. E. Camley, Focusing of spin waves in YIG thin films, IEEE Trans. Magn. 42, 3318 (2006).

[15] V. Veerakumar and R. E. Camley, Magnetostatic bulk and surface spin-wave focusing in antiferromagnetic thin films, Phys. Rev. B 81, 174432 (2010).
[16] F. Heussner, A. A. Serga, T. Brächer, B. Hillebrands, and P. Pirro, A Switchable Spin-Wave Signal Splitter for Magnonic Networks, Appl. Phys. Lett. 111, 122401 (2017).

[17] F. Heussner, M. Nabinger, T. Fischer, T. Brcher, A. A. Serga, B. Hillebrands, and P. Pirro, Frequency-division multiplexing in magnonic logic networks based on caustic-like spin-wave beams, Phys. Status Solidi RRL 12, 1800409 (2018).

[18] F. Heussner, G. Talmelli, M. Geilen, B. Heinz, T. Brcher, T. Meyer, F. Ciubotaru, C. Adelmann, K. Yamamoto, A. A. Serga, B. Hillebrands, and P. Pirro, Experimental realization of a passive gigahertz frequency-division demultiplexer for magnonic logic networks, Phys. Status Solidi RRL 14, 1900695 (2020).

[19] O. Dzyapko, I. V. Borisenko, V. E. Demidov, W. Pernice, and S.O. Demokritov, Reconfigurable heat-induced spin wave lenses, Appl. Phys. Lett. 109, 232407 (2016).

[20] M. van Kampen, C. Jozsa, J. T. Kohlhepp, P. LeClair, L. Lagae, W. J. M. de Jonge, and B. Koopmans, All-Optical Probe of Coherent Spin Waves, Phys. Rev. Lett. 88, 227201 (2002).

[21] A. V. Kimel, A. Kirilyuk, P. A. Usachev, R. V. Pisarev, A. M. Balbashov, and T. Rasing, Ultrafast non-thermal control of magnetization by instantaneous photomagnetic pulses, Nature (London) 435, 655 (2005).

[22] T. Satoh, S.-J. Cho, R. Iida, T. Shimura, K. Kuroda, H. Ueda, Y. Ueda, B. A. Ivanov, F. Nori, and M. Fiebig, Spin Oscillations in Antiferromagnetic NiO Triggered by Circularly Polarized Light, Phys. Rev. Lett. 105, 077402 (2010).

[23] T. Satoh, Y. Terui, R. Moriya, B. A. Ivanov, K. Ando, E. Saitoh, T. Shimura, and K. Kuroda, Directional control of spin-wave emission by spatially shaped light, Nat. Photonics 6, 662 (2012).

[24] Y. Au, M. Dvornik, T. Davison, E. Ahmad, P. S. Keatley, A. Vansteenkiste, B. Van Waeyenberge, and V. V. Kruglyak, Direct Excitation of Propagating Spin Waves by Focused Ultrashort Optical Pulses, Phys. Rev. Lett. 110, 097201 (2013).

[25] S.-J. Yun, C.-G. Cho, and S.-B. Choe, Simultaneous excitation of two different spinwave modes by optical ultrafast demagnetization, Appl. Phys. Express 8, 063009 (2015).

[26] S. Iihama, Y. Sasaki, A. Sugihara, A. Kamimaki, Y. Ando, and S. Mizukami, Quantification of a propagating spinwave-packet created by an ultrashort laser pulse in a thin film of magnetic metal, Phys. Rev. B 94, 020401(R) (2016).

[27] D. Bossini, S. Dal Conte, Y. Hashimoto, A. Secchi, R. V. Pisarev, T. Rasing, G. Cerullo, and A. V. Kimel, Macrospin dynamics in antiferromagnets triggered by sub-20 femtosecond injection of nanomagnons, Nat. Commun. 7, 10645 (2016).

[28] Y. Hashimoto, S. Daimon, R. Iguchi, Y. Oikawa, K. Shen, K. Sato, D. Bossini, Y. Tabuchi, T. Satoh, B. Hillebrands, G. E. W. Bauer, T. H. Johansen, A. Kirilyuk, T. Rasing, and E. Saitoh, All-optical observation and reconstruction of spin wave dispersion, Nat. Commun. 8, 15859 (2017).

[29] A. Kamimaki, S. Iihama, Y. Sasaki, Y. Ando, and S. Mizukami, Reciprocal excitation of propagating spin waves by a laser pulse and their reciprocal mapping in magnetic metal films, Phys. Rev. B 96, 014438 (2017). 
[30] M. Jäckl, V. I. Belotelov, I. A. Akimov, I. V. Savochkin, D. R. Yakovlev, A. K. Zvezdin, and M. Bayer, Magnon Accumulation by Clocked Laser Excitation as Source of Long-Range Spin Waves in Transparent Magnetic Films, Phys. Rev. X 7, 021009 (2017).

[31] Y. Hashimoto, D. Bossini, T. H. Johansen, E. Saitoh, A. Kirilyuk, and T. Rasing, Frequency and wavenumber selective excitation of spin waves through coherent energy transfer from elastic waves, Phys. Rev. B 97, 140404(R) (2018).

[32] S. Muralidhar, A. A. Awad, A. Alemán, R. Khymyn, M. Dvornik, D. Hanstorp, and J. Åkerman, Sustained coherent spin wave emission using frequency combs, Phys. Rev. B 101, 224423 (2020).

[33] A. Kirilyuk, A. V. Kimel, and T. Rasing, Ultrafast optical manipulation of magnetic order, Rev. Mod. Phys. 82, 2731 (2010).

[34] D. Bossini and T. Rasing, Femtosecond optomagnetism in dielectric antiferromagnets, Phys. Scr. 92, 024002 (2017).

[35] I. V. Savochkin, M. Jäckl, V. I. Belotelov, I. A. Akimov, M. A. Kozhaev, D. A. Sylgacheva, A. I. Chernov, A. N. Shaposhnikov, A. R. Prokopov, V. N. Berzhansky, D. R. Yakovlev, A. K. Zvezdin, and M. Bayer, Generation of spin waves by a train of fs-laser pulses: A novel approach for tuning magnon wavelength, Sci. Rep. 7, 5668 (2017).

[36] A. Aleman, S. Muralidhar, A. A. Awad, J. Åkerman, and D. Hanstorp, Frequency comb enhanced Brillouin microscopy, Opt. Express 28, 29540 (2020).

[37] T. Sebastian, K. Schultheiss, B. Obry, B. Hillebrands, and H. Schultheiss, Micro-focused Brillouin light scattering: Imaging spin waves at the nanoscale, Front. Phys. 3, 1 (2015).
[38] M. Dvornik, A. A. Awad, and J. Åkerman, Origin of Magnetization Auto-Oscillations in Constriction-Based Spin Hall Nano-Oscillators, Phys. Rev. Applied 9, 014017 (2018).

[39] A. Awad, P. Dürrenfeld, A. Houshang, M. Dvornik, E. Iacocca, R. Dumas, and J. Åkerman, Long-range mutual synchronization of spin Hall nano-oscillators, Nat. Phys. 13, 292 (2017).

[40] M. Zahedinejad, A. A. Awad, S. Muralidhar, R. Khymyn, H. Fulara, H. Mazraati, M. Dvornik, and J. Åkerman, Twodimensional mutually synchronized spin Hall nano-oscillator arrays for neuromorphic computing, Nat. Nanotechnol. 15, 47 (2020).

[41] C. S. Davies, A. V. Sadovnikov, S. V. Grishin, Y. P. Sharaevskii, S. A. Nikitov, and V. V. Kruglyak, Generation of propagating spin waves from regions of increased dynamic demagnetising field near magnetic antidots, Appl. Phys. Lett. 107, 162401 (2015).

[42] R. Gieniusz, H. Ulrichs, V. D. Bessonov, U. Guzowska, A. I. Stognii, and A. Maziewski, Single antidot as a passive way to create caustic spin-wave beams in yttrium iron garnet films, Appl. Phys. Lett. 102, 102409 (2013).

[43] B. A. Kalinikos and A. N. Slavin, Theory of dipole-exchange spin wave spectrum for ferromagnetic films with mixed exchange boundary conditions, J. Phys. C 19, 7013 (1986).

[44] A. Houshang, R. Khymyn, H. Fulara, A. Gangwar, M. Haidar, S. R. Etesami, R. Ferreira, P. P. Freitas, M. Dvornik, R. K. Dumas, and J. Åkerman, Spin transfer torque driven higher-order propagating spin waves in nano-contact magnetic tunnel junctions, Nat. Commun. 9, 4374 (2018).

[45] K. Niitsu, Temperature dependence of magnetic exchange stiffness in iron and nickel, J. Phys. D 53, 39LT01 (2020). 